\title{
¿Cómo podemos distinguir a los vendedores éticos de los que no lo son?: Implicaciones para el proceso de selección y formación de los comerciales
}

\author{
How can we distinguish ethical salespeople from \\ those who are not?: Implications for the salespeople \\ selection and training process
}

\author{
Sergio Román Nicolás ${ }^{1}$ \\ Rocío RodríGUEz HERrera ${ }^{1}$ \\ Universidad de Murcia (España)
}

Recibido el 21 de diciembre de 2010, aceptado el 28 de septiembre de 2011

$\mathrm{N}^{\circ}$ de clasificación JEL: M12, M39, M40

DOI: $10.5295 /$ cdg. 100273 sr

Resumen:

Actualmente las organizaciones necesitan dar prestigio a sus equipos de ventas, conocedoras de la influencia que tienen los vendedores sobre el éxito o fracaso de la empresa y de la deteriorada imagen que la sociedad ha tenido tradicionalmente de los vendedores, debido en parte, a la utilización de prácticas éticamente cuestionables por parte de los mismos. Nuestro trabajo tiene como principal objetivo analizar el efecto de determinadas variables demográficas (género, formación y edad) y de personalidad (maquiavelismo y materialismo) sobre las evaluaciones que los vendedores hacen de diversos comportamientos de venta éticamente cuestionables. Aplicamos la prueba t-student para diferencia de medias sobre una muestra de 199 comerciales. Los resultados revelan que todas las variables consideradas, excepto el género, influyen significativamente sobre los juicios éticos de los comerciales. Las importantes implicaciones de estos resultados para el proceso de selección y formación del equipo comercial son discutidas al final del artículo.

Palabras clave:

comportamientos de venta éticamente cuestionables; variables demográficas, variables de personalidad, selección de vendedores, formación de vendedores.

\begin{abstract}
:
Nowadays organizations need to improve the image of their salespeople since they are aware of the influence of salespeople behaviors on the success or failure of the company as well as the bad image that society has traditionally had about the sales function, due in part to the use of sales ethically questionable practices. This article aims to analyze the effect of certain demographic variables (gender, formation and age) and personality traits (machiavellianism and materialism) on salespeople's evaluations of sales ethically questionable behaviors. Student t-test of
\end{abstract}

\footnotetext{
${ }^{1}$ Departamento de Comercialización e Investigación de Mercados. Facultad de Economía y Empresa. Universidad de Murcia. Campus de Espinardo.30.100, Espinardo, Murcia.sroman@um.es, rrodriguez@um.es.
} 
difference of means is used on sample of 199 salespeople. The results reveal that all the variables considered, but gender, influenced a salesperson's ethical judgments. The key implications for the sales selection and training activities are discussed at the end of the article.

\section{Keywords:}

ethically questionable sales behaviors, demographic variables, personality variables, sales people selection, salespeople training. 


\section{INTRODUCCIÓN}

A pesar de la vital importancia de la fuerza de ventas para la empresa, tradicionalmente la profesión de vendedor ha estado marginada por la sociedad (Hawes et al., 2004). Sirvan de ejemplo obras de teatro como "Muerte de un viajante" o películas como "Éxito a cualquier precio", donde se muestran personas frustradas y obsesionadas por cerrar la venta aún a costa de los intereses del comprador. Incluso en términos lingüísticos hoy en España se procura evitar la palabra vendedor con la utilización de otros términos como asesores $o$ técnicos comerciales, desarrolladores de negocio, promotores de ventas, representantes, ejecutivos de venta y un largo etcétera. Aunque existe una variada tipología de vendedores, la mala imagen que, en general, se tiene del vendedor, se debe fundamentalmente a dos motivos (1) la infravaloración social en torno a la importancia de la función de la venta personal en la economía y (2) el empleo de malas prácticas comerciales éticamente cuestionables que se han venido utilizando para conseguir la venta del producto sin considerar las necesidades del comprador (p.e. exagerar o mentir sobre los beneficios del producto).

Lo anterior contrasta con el hecho de que hoy en día la mayoría de empresas apuestan por el establecimiento de relaciones a largo plazo con sus clientes. El comportamiento ético y profesional del vendedor ejerce un papel determinante en este sentido, tal y como han puesto de manifiesto diversas investigaciones (Babin et al., 2004; Román y Ruiz, 2005). A pesar de que la ética en la venta es un constructo ambiguo y difícil de delimitar, podemos establecer que el comportamiento ético del vendedor exige actuar de forma justa y sincera, sin presionar y manipular al cliente para que adquiera un producto que no desea (Román y Munuera, 2005).

Existen diversas circunstancias que motivan que los vendedores se encuentren continuamente frente a la toma de decisiones éticas/no éticas en su trabajo: (1) Su labor está sujeta a una menor supervisión por parte de la dirección, al trabajar muchas horas fuera de la empresa; (2) Se enfrentan a una mayor presión frente a otros trabajadores de la empresa, ya que son los principales, sino únicos, responsables de generar ingresos para la organización y además su remuneración y evaluación dependen en muchos casos de los resultados obtenidos.

Partiendo sobre la base de que las evaluaciones o juicios de los vendedores en torno a los comportamientos de venta éticamente cuestionables influyen en su posterior comportamiento (Treviño, 1986; Hunt y Vitell, 1986), el presente trabajo tiene por objetivo analizar la influencia de determinadas variables demográficas (género, edad y formación) y de personalidad (maquiavelismo y materialismo) sobre dichos juicios éticos. Por consiguiente, este trabajo se centra en los juicios o evaluaciones ${ }^{1}$ (opiniones subjetivas) que hacen los vendedores sobre determinados comportamientos de venta no éticos.

\section{VARIABLES QUE INFLUYEN SOBRE LOS JUICIOS ÉTICOS DE LOS VEN- DEDORES}

Existen muy pocos trabajos que hayan analizado empíricamente los determinantes de las evaluaciones/juicios éticos de los vendedores. Henthorne et al. (1992) muestran que

${ }^{1}$ Llamaremos a esta variable indistintamente como juicios o evaluaciones éticas. 
existen diferencias significativas entre las percepciones éticas de los supervisores de venta y sus supervisados; mientras que Lee et al. (2009) ponen de manifiesto que los vendedores de mayor edad son menos flexibles a la hora de juzgar actividades de venta no éticas, pero la experiencia en ventas no tiene un efecto significativo. El trabajo de Ergeneli y Arikan (2002) tampoco encuentra que el género tenga una influencia significativa. Por su parte, Boyle (2000) adopta una perspectiva diferente y descubre que los vendedores juzgan como menos apropiado mentir a los consumidores cuando éstos tienen menos dinero y cuando son mujeres; también halla que los vendedores idealistas son más estrictos a la hora de juzgar los comportamientos de venta no éticos. Este último resultado es consistente con el trabajo posterior de Dubinsky et al. (2004).

Debido a la escasez de estudios y la falta de consistencia en sus resultados, hemos querido revisar la literatura sobre los determinantes de las evaluaciones de los consumidores en torno a las actuaciones éticamente cuestionables de los vendedores. Para facilitar los hallazgos de esta revisión hemos realizado la tabla 1.

Tabla 1

Resumen de estudios que han analizado empíricamente el efecto de diversas variables del individuo (variables independientes) sobre sus juicios éticos (variable dependiente)

\begin{tabular}{|c|c|c|c|}
\hline $\begin{array}{c}\text { VARIABLE } \\
\text { INDEPENDIENTE }\end{array}$ & $\begin{array}{l}\text { TIPO DE } \\
\text { MUESTRA }\end{array}$ & $\begin{array}{c}\text { RESULTADO } \\
\text { SIGNIFICATIVO } \\
\text { (la variable independiente sí } \\
\text { afecta significativamente a } \\
\text { los juicios éticos) }\end{array}$ & $\begin{array}{c}\text { RESULTADO } \\
\text { NO SIGNIFICATIVO } \\
\text { (la variable independiente } \\
\text { no afecta significativamente } \\
\text { a los juicios éticos) }\end{array}$ \\
\hline \multirow{2}{*}{ Edad } & Consumidores & $\begin{array}{l}\text { Volkema (2004) } \\
\text { Ramsey et al. (2007) }\end{array}$ & McIntyre et al. (1999) \\
\hline & Vendedores & Lee et al. (2009) & \\
\hline \multirow[t]{2}{*}{ Género } & Consumidores & $\begin{array}{l}\text { Volkema (2004) } \\
\text { Bellizi y Hasty (1994) }\end{array}$ & $\begin{array}{l}\text { Sparks y Johlke (1996) } \\
\text { McIntyre et al. (1999) }\end{array}$ \\
\hline & Vendedores & & Ergeneli y Arikan (2002) \\
\hline \multirow[t]{2}{*}{ Formación académica } & Consumidores & $\begin{array}{l}\text { McIntyre et al. (1999)1 } \\
\text { Vitell and Muncy (1992) }\end{array}$ & \\
\hline & Vendedores & & \\
\hline \multirow{2}{*}{ Formación en negocios } & Consumidores & Sparks y Johlke (1996) & \\
\hline & Vendedores & & \\
\hline \multirow{2}{*}{ Ingresos } & Consumidores & & McIntyre et al. (1999) \\
\hline & Vendedores & & \\
\hline \multirow{2}{*}{ Idealismo/Relativismo } & Consumidores & $\begin{array}{l}\text { Volkema (2004) } \\
\text { Revilla y Gallego (2007) }\end{array}$ & \\
\hline & Vendedores & $\begin{array}{l}\text { Boyle (2000) } \\
\text { Dubinsky et al. (2004) }\end{array}$ & \\
\hline
\end{tabular}




\begin{tabular}{|c|c|c|c|}
\hline $\begin{array}{c}\text { VARIABLE } \\
\text { INDEPENDIENTE }\end{array}$ & $\begin{array}{l}\text { TIPO DE } \\
\text { MUESTRA }\end{array}$ & $\begin{array}{c}\text { RESULTADO } \\
\text { SIGNIFICATIVO } \\
\text { (la variable independiente sí } \\
\text { afecta significativamente a } \\
\text { los juicios éticos) }\end{array}$ & $\begin{array}{c}\text { RESULTADO } \\
\text { NO SIGNIFICATIVO } \\
\text { (la variable independiente } \\
\text { no afecta significativamente } \\
\text { a los juicios éticos) }\end{array}$ \\
\hline \multirow[t]{2}{*}{ Maquiavelismo } & Consumidores & $\begin{array}{l}\text { Gunnthorsdottir et al. } \\
\text { (2002) }\end{array}$ & \\
\hline & Vendedores & & \\
\hline \multirow{2}{*}{ Materialismo } & Consumidores & Muncy y Eastman $(1998)^{2}$ & \\
\hline & Vendedores & & \\
\hline \multirow{2}{*}{ Experiencia en ventas } & Consumidores & & \\
\hline & Vendedores & & Lee et al. (2009) \\
\hline \multirow{2}{*}{ Nacionalidad } & Consumidores & Li y Persons (2011) & Volkema (2004) \\
\hline & Vendedores & & \\
\hline
\end{tabular}

Fuente: Elaboración propia.

${ }^{l}$ En este caso el resultado significativo es el contrario al planteado en la hipótesis.

${ }^{2}$ En este estudio se analizan los juicios de los consumidores sobre actividades poco éticas de los consumidores

Un análisis rápido de esta tabla pone de manifiesto que, a pesar de la mayor atención prestada a este ámbito de estudio de las evaluaciones de los consumidores, tampoco se ha llegado a resultados concluyentes, siendo habitual que algunos trabajos hayan encontrado resultados significativos, mientras que en otros éstos han sido no significativos. Quizás el ejemplo más notorio estriba en el análisis de la variable género, significativa en los trabajos de Bellizzi y Hasty (1994) y Volkema (2004), pero no significativa en Sparks y Johlke (1996) y McIntyre et al. (1999).

En suma, la revisión de la literatura y la existencia de resultados poco consistentes ponen de manifiesto la conveniencia de profundizar en el estudio de la influencia de las variables demográficas (género, edad y formación) y de personalidad (materialismo y maquiavelismo) en los juicios éticos de los comerciales. A continuación, expondremos las razones teóricas que nos permiten formular el tipo de influencia que estas variables tienen en los juicios éticos de los vendedores.

En primer lugar, podemos basarnos en la teoría de la socialización para explicar las posibles diferencias en los juicios éticos en función del género (Bussey and Maughan, 1982; Gilligan, 1982). Esta teoría establece que fruto del proceso de socialización, en las mujeres adquieren más importancia los valores que se relacionan con la preocupación por otros y el cuidado de los demás; mientras que en los hombres predominan el logro personal y la rivalidad. La preponderancia de unos valores frente a otros provoca, de acuerdo a esta teoría, que los hombres sean más flexibles a la hora de juzgar situaciones o actividades carentes de ética. En cuanto a la edad, la toma de decisiones éticas y las evaluaciones de lo que está bien o mal, en general, aumentan a medida que los individuos se mueven de los niveles inferiores a los niveles superiores de razonamiento moral (Wotruba, 1990) y el razonamiento moral está directamente asociado a la edad (Rest, 1986). Respecto a la 
educación, Kohlberg (1981) en su teoría del desarrollo moral cognitivo, expone que las personas van progresando en su desarrollo moral partiendo de estadios bajos y ascendiendo a lo largo de toda su vida, y que la educación y la formación facilitan a las personas alcanzar estadios superiores en esta evolución. Estos estadios superiores se corresponden con juicios éticos más estrictos en los individuos. En conjunto, los argumentos anteriores nos permiten plantear que:

H1: Los vendedores (a) de género masculino, (b) de menor edad, y (c) sin estudios universitarios serán más tolerantes a la hora de juzgar las situaciones de venta éticamente cuestionables frente a los vendedores de género femenino, de mayor edad y con estudios universitarios.

El maquiavelismo, como rasgo de personalidad, es definido por Robinson y Shaver (1973, p.590) como "un rasgo de la persona para hacer frente al resto de individuos, especialmente cuando la persona siente que puede manejar a los demás". Se trata de un enfoque amoral que ignora las necesidades y derechos de otros, lo que conlleva a los individuos maquiavélicos a utilizar técnicas engañosas o manipuladoras con los demás para beneficio personal (Christie y Geis, 1970). Algunos autores incluso aducen que los individuos maquiavélicos no ven nada malo en robar (Harrell y Hartnagel, 1976), estafar (Flynn et al., 1987) o mentir (Fletcher, 1990) como forma de lograr sus intereses personales (Mudrack, 1993). Por consiguiente, es de esperar que los vendedores más maquiavélicos sean menos estrictos en sus juicios éticos. Es decir:

H2: Los vendedores más maquiavélicos serán más tolerantes con los comportamientos de venta éticamente cuestionables que los vendedores menos maquiavélicos.

Por último, Belk, (1984, p. 291) establece que el materialismo es "la importancia que un individuo otorga a las posesiones mundanas". Se argumenta que los mayores niveles de materialismo conducen a una pérdida del sentido de comunidad, que a su vez puede volver a las personas menos sensibles, ya que éstas se centran en sí mismas y en sus posesiones materiales (Belk, 1988). Esta línea de investigación nos lleva a que los vendedores más materialistas se mostrarán cómodos con las actuaciones poco éticas (por ejemplo, presionar al comprador para vender el producto), porque estas actuaciones aumentan la posibilidad de conseguir metas materiales individuales (la comisión por la venta y por lo tanto la posibilidad de comprar cosas). Por consiguiente planteamos que:

H3: Los vendedores más materialistas serán más tolerantes con los comportamientos de venta éticamente cuestionables que los vendedores menos materialistas.

\section{ESTUDIO EMPÍRICO}

\subsection{Escalas de medición}

En primer lugar, cabe señalar que vamos a emplear la metodología de escenarios para la contrastación de las hipótesis. El empleo de escenarios, donde se plantean y describen situaciones éticamente cuestionables, ha sido ampliamente utilizado en la literatura de ética 
y ventas (p.ej. Bellizzi y Hasty, 1984; Ramsey et al., 2007; Bateman y Valentine, 2010). En concreto, en los escenarios del presente estudio, un consumidor, que en nuestro estudio llamamos Juan, experimenta diversas situaciones de compra en las cuales los comerciales que lo atienden llevan a cabo acciones éticamente cuestionables. Cada escenario fue redactado en tercera persona para transmitir la idea de que los encuestados estaban abordando el comportamiento de otros y no el suyo, siguiendo la línea de otros autores como Ramsey et al. (2007). Los escenarios fueron adaptados principalmente de escenarios creados en otros estudios (Bellizzi y Hasty, 1984; Ramsey et al., 2007; Bateman y Valentine, 2010). También, aunque en menor medida, se han utilizado escenarios nuevos en los que los autores de este trabajo nos hemos visto envueltos (véase la tabla 2). Al final de cada uno se le pedía al encuestado que manifestara su grado de acuerdo / desacuerdo con la siguiente afirmación: "El comportamiento de este vendedor es apropiado", siendo 1= "Totalmente en desacuerdo y $7=$ "Totalmente de acuerdo".

Tabla 2

Descripción de los escenarios (media y desviación típica entre paréntesis)

Juan tiene una boda y acude a una tienda para comprarse un traje nuevo. Tras pro-

Escenario 1 barse uno, cuyo precio es razonable, el dependiente le comenta que el traje parece

$(2.95 ; 1.64)$ "hecho a su medida". La estrategia de este dependiente es decirle a todos sus clientes, que la ropa les "queda fenomenal", independientemente de que sea así o no

Juan quiere comprarse un ordenador con una buena tarjeta gráfica y acude a una tienda de ordenadores. Juan no sabe nada de ordenadores por lo que espera, que el Escenario 2 dependiente le asesore y le ofrezca las características, que está pidiendo al mejor $(1.88 ; 1.30) \quad$ precio. Sin embargo, el dependiente tiene que "dar salida" a unos ordenadores, que no se están vendiendo bien y cuya tarjeta gráfica no es buena. Por lo tanto, el dependiente le oferta a Juan este ordenador sin decirle nada sobre la tarjeta gráfica.

Posteriormente, Juan va a otra tienda de ordenadores. Necesita una impresora y le Escenario 3 pregunta al dependiente, si una de ellas es compatible con su equipo. El vendedor, $(1.85 ; 1.17) \quad$ sin prestarle mucha atención, le dice que sí, que es compatible con su ordenador.

Juan tiene que comprar un router y acude a una tercera tienda de ordenadores.

Escenario 4 Los routers este mes tienen un $20 \%$ de descuento, para todos los clientes. Sin $(2.67 ; 1.52)$ embargo, el vendedor le dice a Juan que le hace un $20 \%$ de descuento en el router, porque "es un buen cliente", ya que le ha comprado previamente.

Juan se va de viaje en una semana y acaba de darse cuenta de que su tarjeta de

Escenario 5 crédito ha caducado. Acude a su oficina bancaria y contrata una nueva tarjeta con mayor crédito (una visa oro). El de la oficina le asegura, que la tarjeta estará en su casa en menos de una semana, aunque sabe que algunas veces la central ha tardado más tiempo en enviarla.

Juan quiere comprarle un perfume a su hermana. Le resulta indiferente comprar Escenario 6 uno u otro. En la tienda se decide por uno que le ofrece el vendedor, porque es $(2.74 ; 1.71) \quad$ "una fragancia única". El vendedor no le ha dicho, que el perfume entra en una campaña del promoción del $25 \%$ al día siguiente.

Fuente: Elaboración propia. 
Maquiavelismo y materialismo se han medido con escalas ampliamente utilizadas en la literatura y suficientemente validadas. En concreto, para el maquiavelismo se han utilizado 4 ítems de Christie et al. (1970). Estudios previos han empleado satisfactoriamente versiones reducidas de esta escala (Allsop et al., 1991; Rawwas et al., 2005). Se utilizaron 4 ítems de la escala de Richins y Dawson (1992) para medir el materialismo. Estudios previos también han empleado versiones reducidas de esta escala (Keng et al., 2000). En todos los casos se ha empleado una escala tipo Likert de 7 puntos siendo $1=$ "Totalmente en desacuerdo y $7=$ "Totalmente de acuerdo".

Tabla 3

\section{Escalas de medida adaptadas}

\begin{tabular}{|c|c|c|}
\hline Medida & Ítems (media y desviación típica entre paréntesis) & Escala \\
\hline \multirow{4}{*}{ Maquiavelismo } & $\begin{array}{l}\text { Para salir adelante hay que "hacer la pelota" a las personas que } \\
\text { nos interesan }(3.22 ; 1.46) .\end{array}$ & \multirow{4}{*}{$\begin{array}{l}\text { Christie et } \\
\text { al. (1970) }\end{array}$} \\
\hline & $\begin{array}{l}\text { Sólo cuando me beneficia digo los verdaderos motivos por los } \\
\text { que hago algo }(2.97 ; 1.33) \text {. }\end{array}$ & \\
\hline & $\begin{array}{l}\text { Para progresar en la vida hay que elegir el camino más corto } \\
(2.61 ; 1.33)\end{array}$ & \\
\hline & $\begin{array}{l}\text { La mejor forma de relacionarte con los demás es decirles lo que } \\
\text { quieren oír }(2.85 ; 1.50)\end{array}$ & \\
\hline \multirow{4}{*}{ Materialismo } & $\begin{array}{l}\text { Admiro a las personas que tienen buenas casas, coches, etc. } \\
(3.69 ; 1.53)\end{array}$ & \multirow{4}{*}{$\begin{array}{l}\text { Richins y } \\
\text { Dawson } \\
(1992)\end{array}$} \\
\hline & $\begin{array}{l}\text { Mis posesiones dicen mucho de lo que he conseguido en la vida } \\
(3.48 ; 1.52)\end{array}$ & \\
\hline & $\begin{array}{l}\text { Es importante adquirir bienes materiales para tener éxito en la } \\
\text { vida }(2.84 ; 1.40)\end{array}$ & \\
\hline & Me gusta tener cosas que impresionen a la gente $(3.03 ; 1.51)$ & \\
\hline
\end{tabular}

Realizamos un análisis factorial exploratorio con rotación varimax sobre las tres variables multi-ítem. Los resultados demostraron que cada factor cargaba en su variable correspondiente. Entonces calculamos el valor del Alpha de Cronbach para cada una de las escalas, siendo, 0.72 para el maquiavelismo, 0.84 para el materialismo, y 0.72 para la escala de evaluación del vendedor en torno a los comportamientos de venta éticamente cuestionables. Todos los valores son satisfactorios de acuerdo con Nunnally (1978).

Finalmente se estimó un modelo de medida estructural (LISREL 8.80) con las tres variables multi-item (juicios éticos, maquiavelismo y materialismo) para testar la validez convergente y discriminante de las escalas. Los resultados del modelo son buenos $\left(\chi^{2}(74)=117.65 \mathrm{p}<0.01 ; \mathrm{CFI}=0.96 ; \mathrm{NNFI}=0.95 ; \mathrm{GFI}=0.92 ; \mathrm{RMSEA}=0.05 ; \mathrm{RMSR}=0.05\right)$. El valor de los coeficientes estandarizados (lambda) de cada indicador y su alta significatividad (el valor de la $t$ más bajo es de 6.16) ponen de manifiesto la validez convergente 
de las escalas (Anderson y Gerbing, 1988). Por otra parte, en todos los casos el intervalo de confianza (+/-2 veces el error estándar) de la correlación estimada entre dos variables ( $\Phi)$ no incluía el valor uno, lo que permite establecer la existencia de validez discriminante entre las escalas. Es decir, aunque relacionadas, las variables de materialismo, maquiavelismo y juicio ético miden constructos distintos.

\subsection{Recogida de datos y descripción de la muestra.}

En una primera etapa se realizó un pretest con comerciales para mejorar la redacción de los ítems y asegurarnos que comprendían los aspectos que queríamos medir. El reciente trabajo de Carter et al. (2008), que analiza 532 muestras de estudios realizados entre 1990 y 2005 en el ámbito de la venta personal y la dirección de ventas, pone de manifiesto la creciente dificultad para obtener datos primarios de muestras de vendedores y sugieren emplear nuevas formas de recogida de información que sean flexibles y rápidas. En concreto, los autores finalizan su estudio animando a los investigadores de ventas a utilizar muestras de conveniencia para aumentar el índice de respuesta (Carter et al., 2008, p.414). Este hecho, unido a la sensibilidad social de los temas tratados (por ejemplo, el grado en el que el vendedor manifiesta ser maquiavélico), determinó que optáramos por un muestreo no probabilístico o de conveniencia con la creación de una página web donde el cuestionario estaba insertado. De esta manera, se facilitaba su cumplimentación así como el anonimato y confidencialidad de la información aportada por los vendedores. En la recogida de datos seguimos un procedimiento similar a estudios anteriores (p.e. Best y Krueger, 2002 y De Bruyn et al., 2008). Inicialmente se utilizaron listas de correo electrónicos de amigos, conocidos y familiares de los autores, así como de diversas organizaciones y empresas. En el correo se invitaba a los sujetos a participar en el estudio, siempre que fueran comerciales, y también a que le enviaran el correo con la dirección de la página web a otros comerciales que conocieran.

La muestra final de cuestionarios completos está compuesta por 199 comerciales. La forma de administrar el cuestionario nos impide proporcionar una tasa de respuesta. Las características demográficas de la muestra aparecen recogidas en la tabla 4, donde observamos que existe un mayor porcentaje de hombres que se dedican a la venta 63,3\% y la edad media de los encuestados es de 34 años. En cuanto a los estudios, son los universitarios los que tienen mayor porcentaje muestral con un 70,4\%. Poco más del 50\% de la muestra afirma tener un nivel de ingresos familiares medio (de $1000 €$ a $3000 €$ ).

Tabla 4

\begin{tabular}{clc}
\hline & Características demográficas de la muestra $(\mathrm{n}=199)$ & Porcentaje $(\%)$ \\
\hline \multirow{2}{*}{ Género } & Hombre & 63,3 \\
\cline { 2 - 3 } & Mujer & 36,7 \\
\hline \multirow{2}{*}{ Edad } & Menor de 34 & 46,2 \\
\cline { 2 - 3 } & Mayor de 34 & 53,8 \\
\hline
\end{tabular}




\begin{tabular}{llc}
\hline & Características demográficas de la muestra $(\mathrm{n}=199)$ & Porcentaje $(\%)$ \\
\hline \multirow{3}{*}{ Estudios } & Sin estudios universitarios & 29,6 \\
\cline { 2 - 3 } & Universitarios & 70,4 \\
\hline \multirow{3}{*}{ Ingresos } & Bajos & 6 \\
\cline { 2 - 3 } & Medios & 51,7 \\
\cline { 2 - 3 } & Altos & 42,3 \\
\hline
\end{tabular}

\section{RESULTADOS}

Se ha utilizado la prueba $t$ de Student de diferencia de medias para muestras independientes para analizar la influencia de las variables demográficas y de personalidad en las evaluaciones del vendedor sobre comportamientos de venta éticamente cuestionables (véase la tabla 5). Es decir, la variable a contrastar es la suma aritmética de los seis escenarios planteados descritos en la tabla 2. Las variables agrupación han sido divididas en las siguientes categorías: género (hombres vs. mujeres), edad (vendedores menores 34 años vs. vendedores mayores de 34 años), formación (vendedores sin estudios universitarios vs. con estudios universitarios), maquiavelismo (vendedores menos maquiavélicos vs. más maquiavélicos) y materialismo (vendedores menos materialistas vs. más materialistas). A excepción de la variable formación, el resto fueron dividas por la mediana en consonancia con investigaciones similares (Altemeyer, 2004).

Tabla 5

\section{Resumen de Resultados}

\begin{tabular}{|c|c|c|c|c|c|c|}
\hline \multicolumn{7}{|c|}{ Diferencia de medias: Prueba t-student } \\
\hline $\begin{array}{l}\text { Variable de } \\
\text { agrupación }\end{array}$ & Categorías & $\mathrm{N}$ & Media $^{1}$ & $t$ & Sig. & $\begin{array}{c}\text { Tamaño del } \\
\text { efecto }(d)\end{array}$ \\
\hline \multirow{2}{*}{ Género } & Hombre & 126 & 2,10 & \multirow{2}{*}{$-0,62$} & \multirow{2}{*}{ n.s. } & \multirow{2}{*}{ no aplicable } \\
\hline & Mujer & 73 & 2,19 & & & \\
\hline \multirow{2}{*}{ Edad } & Menor de 34 & 92 & 2,27 & \multirow{2}{*}{2,39} & \multirow{2}{*}{0,018} & \multirow{2}{*}{0,34} \\
\hline & Mayor de 34 & 107 & 2,00 & & & \\
\hline \multirow[t]{2}{*}{ Estudios } & $\begin{array}{l}\text { Sin estudios } \\
\text { universitarios }\end{array}$ & 59 & 2,30 & \multirow[t]{2}{*}{1,80} & \multirow[t]{2}{*}{0,073} & \multirow[t]{2}{*}{0,27} \\
\hline & Universitarios & 140 & 2,06 & & & \\
\hline \multirow{2}{*}{ Maquiavelismo } & Bajo & 94 & 1,87 & \multirow{2}{*}{$-2,60$} & \multirow{2}{*}{0,010} & \multirow{2}{*}{0,62} \\
\hline & Alto & 105 & 2,36 & & & \\
\hline \multirow{2}{*}{ Materialismo } & Bajo & 92 & 1,97 & \multirow{2}{*}{$-2,60$} & \multirow{2}{*}{0,010} & \multirow{2}{*}{0,36} \\
\hline & Alto & 107 & 2,27 & & & \\
\hline
\end{tabular}

n.s.= no significativo

${ }^{1}$ Se trata de la media aritmética de los 6 escenarios que por consiguiente oscila entre 1 y 7 .

En la columna del tamaño el efecto: Pequeño $(0<\mathrm{d}<0,20)$; Mediano $(0,21<\mathrm{d}<0,50)$ y Grande $(d>0,51)$. 
Los resultados del análisis de datos se reflejan en la tabla 5, donde se observa que todas las hipótesis planteadas se cumplen satisfactoriamente a excepción de H1a, que establecía diferencias por género. El tamaño del efecto es grande $(0,62)$ para el caso del maquiavelismo, y mediano para el resto (obsérvese la última columna de la tabla 5).

\section{DISCUSIÓN Y CONCLUSIONES}

Pensamos que en este apartado de discusión debemos dedicar una mayor atención a reflexionar sobre el hecho de que en nuestro estudio el género del comercial no influye sobre sus juicios éticos. Una primera explicación podría ser que la teoría sobre la que hemos basado esta hipótesis (teoría de la socialización) se desarrolló por psicólogos y sociólogos sobre los roles de las mujeres y hombres sin considerar su profesión; mientras que nosotros la hemos aplicado sobre una muestra de comerciales, cuyo desempeño laboral, como poníamos de manifiesto al comienzo de este artículo tiene unas particularidades distintivas frente a otras profesiones. Estas características (trabajar fuera de la empresa, trabajar generalmente solo, estar sujeto a una fuerte presión para conseguir objetivos) han podido afectar al resultado no significativo hallado. En segundo lugar, es posible que la teoría de la socialización, independientemente de la profesión que consideremos, tenga una limitada aplicación en la actualidad. En concreto, han pasado casi treinta años desde que se desarrollara esta teoría. En este periodo la creciente incorporación de la mujer al mundo laboral ha podido provocar que las diferencias en cuanto al proceso de socialización y los roles de los géneros tiendan a minimizarse, pudiendo, por lo tanto, explicar que ambos otorguen la misma importancia a los aspectos de la ética en la venta.

Respecto a los resultados significativos, en línea con el trabajo de Lee et al. (2009), encontramos que los vendedores de mayor edad perciben una mayor gravedad en los comportamientos de venta éticamente cuestionables. Al margen de ser consistente con la teoría del desarrollo moral, en el mundo de la venta tal efecto puede producirse también porque normalmente el personal de ventas más joven e inexperto está sujeto a una mayor presión para cumplir con los objetivos y debe realizar un mayor número de visitas a "puerta fría" en las que frecuentemente se aplican técnicas de venta a presión. Por su parte, los vendedores con mayor formación son más críticos a la hora de juzgar los comportamientos de venta éticamente cuestionables, esto se debe a que la educación facilita el desarrollo moral del individuo.

Los vendedores con un maquiavelismo y materialismo altos son menos críticos a la hora de juzgar los comportamientos de venta éticamente cuestionables. Estos hallazgos son muy importantes para la literatura de dirección de ventas pues la evidencia empírica anterior en este sentido se había hallado exclusivamente con muestras de consumidores y no con vendedores tal y como se puso de manifiesto en la tabla 1.

En resumen, los resultados muestran cómo los vendedores de menor edad, menos formados, más maquiavélicos y materialistas son menos críticos/más permisivos a la hora de juzgar los comportamientos de venta éticamente cuestionables. Nuestro trabajo añade claridad a los resultados inconsistentes hallados previamente en la literatura. No obstante, hemos considerado tres variables demográficas y dos de personalidad, por lo que futuros estudios deben ampliar el espectro de variables que pueden influir en los juicios éticos de 
los vendedores. Asimismo, a pesar de que los investigadores en ventas aconsejan el uso de muestras de conveniencia para aumentar el índice de respuesta, las conclusiones que se derivan de este estudio deben interpretarse con cautela en tanto que la muestra obtenida en este trabajo no es aleatoria y por consiguiente, recomendamos testar estas relaciones con otras muestras aleatorias de vendedores.

\section{IMPLICACIONES PARA LAS EMPRESAS}

En el entorno actual donde la mayor competitividad, las nuevas tecnologías, la globalización de la economía y la creciente preparación y formación de los consumidores obligan a las empresas a diferenciar su oferta, a mostrarse responsables con la sociedad (Ramos y Periáñez, 2003), un mayor enfoque ético y profesional del personal de ventas supone una diferenciación en la comercialización del producto, y además conlleva, a una mayor satisfacción del mismo y al establecimiento de una relación entre las partes, como han puesto de manifiesto diversos trabajos (Babin et al., 2004; Román y Ruiz, 2005). Por lo tanto, dado que el juicio ético es uno de los principales antecedentes del comportamiento posterior del vendedor, este estudio tiene importantes implicaciones para la dirección de ventas que quiera fomentar el enfoque ético en su equipo a través de las políticas de selección y formación.

Actualmente en los procesos de selección del personal de ventas se utilizan como herramientas diversos tests como: BAC (Batería para la Actividad Comercial), NEGO (Test de habilidades de negociación), SIV (Cuestionario de valores interpersonales), TAV (Test de Apreciación de Vendedores), o algunos otros más conocidos como son el IPV (Inventario de Personalidad para Vendedores), y el 16 PF. Resulta conveniente incidir en que la mayoría de ellos fueron desarrollados hace más de tres décadas y no tienen en cuenta la nueva función de la fuerza de ventas donde se potencia un enfoque ético y profesional. De hecho, ninguno de los tests citados con anterioridad miden las variables de maquiavelismo y materialismo, que como hemos demostrado afectan a las percepciones de los vendedores sobre las situaciones éticamente cuestionables. Proponemos, por tanto, añadir a las pruebas realizadas en la selección del personal de ventas, los ítems de materialismo y maquiavelismo depurados y adaptados de las escalas norteamericanas que han sido utilizados en esta investigación (véase la tabla 3). Asimismo, recomendamos a la dirección de ventas que quiera enfatizar el enfoque ético en su equipo seleccionar a personal con formación académica y no excesivamente joven.

Por su parte, las actividades formativas también deben perseguir que el personal de ventas adquiera las suficientes y apropiadas habilidades y conocimientos para comportarse de forma ética. Para ello, resulta conveniente que la dirección ponga en práctica la formación a través de ejercicios de simulación que representen distintas situaciones de venta, y donde los vendedores desempeñan consecutivamente ambos roles, tanto el del cliente como el del vendedor. En este sentido, las empresas pueden utilizar y ampliar los escenarios que hemos elaborado en el presente trabajo. Se trata de escenarios que presentan situaciones éticamente cuestionables. Una vez que se ha realizado el ejercicio de rol-play, el formador deberá fomentar la discusión sobre las opiniones de los vendedores en torno a la simulación realizada y explicar, en última instancia, por qué se trata de situaciones poco 
éticas y cómo se deberían hacer de una forma ética y profesional. Por último, de nuestros hallazgos también se deriva que la formación sobre aspectos éticos debe ser especialmente importante en aquellos vendedores que tienen una menor formación académica y son más jóvenes. En estos casos, la formación teórica presencial impartida debe ser reforzada con sencillos vídeos (que el trabajador puede ver en su casa), donde se ejemplifiquen pautas de actuación ante situaciones de venta no éticas. También, los vendedores expertos, con mayor formación y de más edad, podrán acompañar en las visitas a los más jóvenes y con menor formación y, con posterioridad asesorarlos sobre cómo mejorar su enfoque ético con los compradores.

\section{REFERENCIAS BIBLIOGRÁFICAS}

ALLSOP, J., EYSENCK, H. J. y EYSENCK, S. B. G. (1991): "Machiavellianism as a Component in Psychoticism and Extraversion", Personality and Individual Differences, Vol. 12, pp. 29-41.

ALTEMEYER, B. (2004): "Highly Dominating, Highly Authoritarian Personalities", The Journal of Social Psychology, Vol.144, pp. 421-447.

BABIN, B. J., GRIFFIN, M. y BOLES, J. S. (2004): "Buyer Reactions to Ethical Beliefs in the Retail Environment", Journal of Business Research, Vol. 57, pp. 1155-1163.

BATEMAN, C. y VALENTINE, S. (2010): “Investigating the Effects of Gender on Consumers' Moral Philosophies and Ethical Intentions”, Journal of Business Ethics, Vol. 95, pp. 393-404.

BELLIZZI, J.A. y HASTY, R.W. (1998): "Student Perceptions of Questionable Personal Selling Practices", Journal of the Academy of Marketing Science, Vol. 12(1/2), pp. 218-226.

BELK, R.W. (1984): "Cultural and Historical Differences in Concept of Self and their Effects on Attitudes towards Having and Living", Advances in Consumer Research, Vol. 11, pp. 753-760.

BELK, R.W. (1988): "Third World Consumer Culture", Research in Marketing, Vol. (Suppl.4), pp. 102-107.

BEST, S.J. y KRUEGER, B. (2002): "New Approaches to Assessing Opinion: The Prospects for Electronic Mail Surveys", International Journal of Public Opinion Research, Vol. 14, pp. 73-91.

BOYLE, B. (2000):"The Impact of Customer Characteristics and Moral Philosophies on Ethical Judgments of Salespeople", Journal of Business Ethics, Vol. 3, pp. 249-267.

BUSSEY, K. y MAUGHAM, B. (1982):"Gender Differences in Moral Reasoning", Journal of Personality and Social Psychology, Vol. 42, pp. 701-706.

CARTER, R.E., DIXON, A.L. y MONCRIEF, W.C. (2008): “The Complexities of Sales and Sales Management Research: a Historical Analysis from 1990 to 2005”, Journal of Personal Selling \& Sales Management, Vol. 28 (4), pp. 403-419.

CHRISTIE, R. y GEIS, F.L. (1970): "Studies in Machiavellianism", Academic Press, New York.

DE BRUYN, A., LIECHTY, J.L., HUIZINGH, E.K.R.E. y LILIEN, G.L. (2008): “Offering Online Recommendations with Minimum Customer Input through Conjoint-Based Decision Aids", Marketing Science, Vol. 27(3), pp. 443-464.

DUBINSKY, A. NATARAAJAN, R. y HUANG, W.Y. (2004):'The Influence of Moral Philosophy on Retail Salespeople's Ethical Perceptions”, Journal of Consumer Affairs, Vol. 38, pp. 297-319.

ERGENELI, A. y ARIKAN, S. (2002): "Gender Differences in Ethical Perception of Salespeople: An Empirical Examination in Turkey”, Journal of Business Ethics, Vol. 40, pp. 247-260.

FLETCHER, C. (1990): “The Relationship between Candidate Personality, Self-Presentation Strategies, and Interviewer Assessments in Selection Interviews: An Empirical Study”, Human Relations, Vol. 43, pp. 739-749.

FLYNN, S., REICHARD, M. y SLANE, S. (1987): "Cheating as a Function of Task Outcome and Machiavellianism”, Journal of Psychology, Vol. 121, pp. 423-427. 
GERRARD, M., ANGLIN, K.A., SZYMANSKI, D.M. y GENTRY, J.W. (1992): "Relationship Development in Selling: A Cognitive Analysis", Journal of Personal Selling and Sales Management, Vol.12, pp. 23-34.

GILLIGAN, C. (1982): “In a different voice: Psychological theory and women's development”, MA: Harvard University Press, Cambridge.

GUNNTHORSDOTTIR, A., MCCABE, K. y SMITH, V. (2002): "Using the Machiavellianism Instrument to Predict Trustworthiness in a Bargaining Game", Journal of Economic Psychology, Vol. 23, pp. 49-66.

HARRELL, W.A. y HARTNAGEL, T. (1976): "The impact of Machiavellianism and the trustfulness of the victim on laboratory theft", Sociometry, Vol. 39, pp. 157-165.

HAWES, J. RICH, A.K. y WIDMIER, S.M., (2004): "Assessing The Development of The Sales Profession", Journal of Personal Selling \& Sales Management, Vol. 24 (1), pp. 27-34.

HENTHORNE, T.L., ROBIN, D.P. y REIDENBACH, R.E. (1992): "Identifying the Gaps in Ethical Perceptions between Managers and Salespersons", Journal of Business Ethics; Vol.11, pp. 849862.

HUNT, S.D. y VITELL, S.J. (1986): “A General Theory of Marketing Ethics”, Journal of Macromarketing, Vol. 6, pp. 5-16.

KENG, K.A., KWON, J., TAN S.J. y JOCHEN, W. (2000): "The Influence of Materialistic Inclination on Values, Life Satisfaction, and Aspirations: An Empirical Analysis", Social Indicators Research, Vol. 49, pp. 317-333.

KOHLBERG, L. (1981): "Essays on Moral Development", The Philosophy of Moral Development, San Francisco, Vol. 1, CA: Harper \& Row.

LEE, N., BEATSON, A., GARRETT, T., LINGS, I. y ZHANG, X. (2009): "A study of the attitudes towards unethical selling amongst Chinese salespeople", Journal of Business Ethics, Vol. 88, pp. 497-515.

LI, S.F. y PERSONS, O.S. (2011): "Cultural Effects on Business Students' Ethical Decisions: A Chinese Versus American Comparison”, Journal of Education for Business, Vol. 86, pp. 10-16.

MCINTYRE, F.S., THOMAS J.L. y GILBERT, F.W. (1999): "Consumer Segments and Perceptions of Retail Ethics", Journal of Marketing Theory and Practice, Vol. 2, pp. 43-53.

MUDRACK, P. (1993): "Are the Elderly Really Machiavellian? A Reinterpretation of an Unexpected Finding", Journal of Business Ethics, Vol. 14, pp. 757-758.

MUNCY, J.A. y EASTMAN, J.K. (1998): "Materialism and Consumer Ethics: an Exploratory Study", Journal of Business Ethics, Vol. 17, pp. 137-45.

NUNNALLY, J.C. (1978): Psycometric Theory, McGraw-Hill, New York, 2nd ed.

RAMOS, J.A. y PERIAÑEZ (2003):“Delimitación del Marketing con Causa o Marketing Social Corporativo mediante el Análisis de Empresas que Realizan Acciones de Responsabilidad Social", Cuadernos de Gestión, Vol. 3. № 1 y No 2, pp.65-82.

RAMSEY, R.P., MARSHALL, G.W., JOHNSTON, M.W y DEETER-SCHMELZ, D.R. (2007): "Ethical Ideologies and Older Consumer Perceptions of Unethical Sales Tactics", Journal of Business Ethics, Vol. 70, pp. 191-207.

RAWWAS, M.Y.A., SWAIDAN, Z. y OYMAN M. (2005): "Consumer Ethics: A Cross-Cultural Study of the Ethical Beliefs of Turkish and American Consumers", Journal of Business Ethics, Vol. 57(2), pp. 183-195.

REST, J.R. (1986): "Moral Development: Advances in Research and Theory", Praeger, NY.

REVILLA, C. y GALLEGO, Á. (2007): "La Importancia de la Ética en la Venta desde el Punto de Vista del Consumidor", Investigaciones Europeas de Dirección y Economía de la Empresa, Vol. 13, pp. 209-230.

RICHINS, M.L. y DAWSON, S.A. (1992): "Consumer Values Orientation for Materialism and Its Measurement: Scale Development and Validation", Journal of Consumer Research, Vol. 19(3),pp. 303-316 
ROBINSON, J.P. y SHAVER, P.R. (1973): Measures of Social Psychological Attitudes, MI: Institute for Social Research, Ed. Ann Arbor.

ROMÁN, S. y MUNUERA, J.L. (2005): "Determinants and Consequences of Ethical Behaviour: An Empirical Study on Salespeople", European Journal of Marketing, Vol. 39, pp. 473-495.

ROMÁN, S. y RUIZ, S. (2005): "Relationship Outcomes of Perceived Ethical Sales Behavior: the Customer's Perspectives", Journal Business Research, Vol. 58, pp. 439-445.

SPARKS, J.R. y M. JOHLKE (1996): "Factors Influencing Student Perceptions of Unethical Behavior by Personal Salespeople: An Experimental Investigation", Journal of Business Ethics, Vol. 15 , pp. 871-887.

TREVIÑO, L.K. (1986): "Ethical Decision Making in Organizations: A Person-Situation Interactionist Model", Academy of Management Review, Vol. 11, pp. 601-617.

VITELL, S.J. y MUNCY, J. (1992): "Consumer Ethics: An Empirical Investigation of Factors Influencing Ethical Judgments of the Final Consumer", Journal of Business Ethics, Vol.11, pp. 585-597.

VOLKEMA, R.J. (2004): "Demographic, Cultural, and Economic Predictors of Perceived Ethicality of Negotiation Behavior: A Nine-Country Analysis", Journal of Business Research, Vol. 57, pp. 69-78.

WOTRUBA, T. (1990): "A Comprehensive Framework for the Analysis of Ethical Behavior, with a Focus on Sales Organisations", Journal of Personal Selling \& Sales Management, Vol. 10, pp. $29-42$. 
